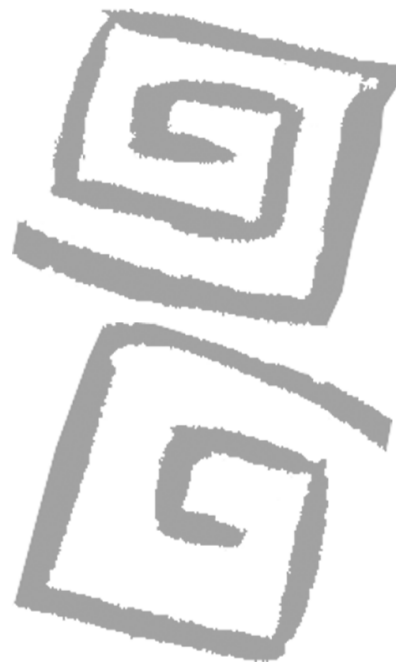
Noronha, Ilma Horsth ${ }^{3}$

\section{El acceso a la información como determinante social de la salud}

\author{
Information access as a social \\ determinant of health
}

${ }^{1}$ Doctora en Ciencias de la Información. Investigadora en Salud Pública, Instituto de Comunicação e Informação Científica e Tecnológica em Saúde (ICICT), Fundação Oswaldo Cruz (Fiocruz), Brasil. cguima@icict.fiocruz.br

${ }^{2}$ Doctora en Ciencias de la Información y de la Comunicación.

Investigadora en Salud Pública, Instituto de Comunicação e Informação Científica e Tecnológica em Saúde (ICICT), Fundação Oswaldo Cruz (Fiocruz), Brasil.

chenrique@icict.fiocruz.br

${ }^{3}$ Magíster en Ciencias de la Información. Coordinadora de la Rede de Bibliotecas da Fiocruz, Instituto de Comunicação e Informação Científica e Tecnológica em Saúde (ICICT), Fundação Oswaldo Cruz (Fiocruz), Brasil.

ilma@icict.fiocruz
Guimarães, Maria Cristina Soares ${ }^{1}$; Silva, Cícera Henrique²;

RESUMEN A lo largo de más de un siglo de investigación, permanece viva la discusión sobre las dimensiones formativas y explicativas que tienen los procesos sociales con relación a la salud de las poblaciones. Las evidencias recogidas a lo largo de este período, en los más distintos contextos y tiempos históricos, apuntan hacia una ecuación compleja entre salud, desigualdades en las condiciones de vida y la red de vínculos y asociaciones entre individuos y grupos. Por ser evitables, injustas e innecesarias, las inequidades en salud son elementos susceptibles de intervención; en particular, el acceso a la información emerge como una dimensión importante para la equidad en salud. Este artículo presenta una discusión inicial sobre el concepto de acceso a la información y de algunos de los distintos marcos y dimensiones en el ámbito de la salud. Finaliza con la propuesta de construcción de una agenda orientada a la promoción del uso de la información en y para la salud.

PALABRAS CLAVE Acceso a la Información; Desigualdades en la Salud; Alfabetización en Salud; Tecnología de la Información.

ABSTRACT After more than a century of research, the debate about how social processes shape the health of populations remains very much alive. The evidence collected througout this time, in its variety of contexts and historical periods, points to a complex equation between health, inequalities in living conditions and the interactions and associations between individuals and groups. Because they are avoidable, unfair and unnecessary, health inequities are elements open to intervention: in particular, access to information emerges as an important dimension of health equity. This article aims to contribute to the discussion of this topic, especially with regard to the concept of access to information and some of its various dimensions. Finally, the article proposes the need for launching an affirmative agenda focused on promoting the use of information in and for health.

KEY WORDS Access to Information; Health Inequalities; Health Literacy; Information Technology. 


\section{INTRODUCCIÓN}

La creciente importancia de los estudios acerca de las inequidades en salud, es decir, de aquellas desigualdades entre grupos de poblaciones que, además de sistemáticas y relevantes, son también injustas, evitables e innecesarias (1), atestiguan las limitaciones del punto de vista del riesgo individual como factor explicativo para el análisis de la salud de las poblaciones (2). El principal desafío del estudio de las relaciones entre determinantes sociales y salud es establecer una jerarquía entre los factores más generales, de naturaleza social, económica y política, y las mediaciones a través de las cuales esos factores se reflejan sobre la situación de grupos y personas, que están lejos de ser una relación simple y directa de causa y efecto (3).

Hay tres generaciones de estudios sobre las inequidades en salud (4). La primera se dedicó a describir las relaciones entre pobreza y salud. La segunda tuvo su foco en los gradientes de salud de acuerdo con varios criterios de estratificación socioeconómica. El esfuerzo actual de investigación, que marca la tercera generación de estos estudios, estaría volcado a identificar y discutir los mecanismos de producción de las inequidades en salud. Esas causas sociales fundamentales involucran recursos tales como conocimiento, poder, dinero, conexiones sociales y prestigio que moldean intensamente la capacidad de las personas de prevenir y reducir al mínimo las consecuencias de la eventual exposición al riesgo y la enfermedad (5) y, a su vez, esos recursos forjan múltiples relaciones con distintos factores de riesgo.

Los tres principales enfoques y respectivos modelos que buscan explicitar las distintas dimensiones involucradas en los determinantes sociales de salud (DSS) son: el enfoque que se centra en los factores psicosociales, el centralizado en la producción social de la enfermedad y el que apunta a la ecología y otros marcos multiniveles (6). Todos los modelos de DSS comparten tres características: reconocen la importancia del contexto; la capacidad del ambiente social, económico, cultural y físico para cambiar la relación entre la salud y las características individuales; y reconocen, aunque no desvelen, la complejidad de las interacciones entre varios determinantes.

Ese complejo de mediaciones es lo que permite explicar, por ejemplo, el límite de las relaciones entre indicadores de riqueza y de salud de una sociedad. Evidencias recientes indican que, cuando es superado un determinado nivel del PIB per cápita, el factor más importante para explicar la situación general de la salud de un determinado país no es su riqueza total, sino la forma como ella se distribuye (7); o bien, que lo importante es distinguir los factores sociales que influyen sobre la salud y los procesos que determinan su distribución desigual.

Otra dimensión significativa indicada por los estudios recientes habla del papel de las redes sociales y comunitarias en los DSS y el papel de movilización de las relaciones de solidaridad y confianza entre personas y grupos. Ese capital social es, por ejemplo, un importante mecanismo por medio del cual las desigualdades en el ingreso tienen un impacto negativo sobre la situación de salud. La debilidad de los lazos de cohesión ocasionada por las inequidades en el ingreso corresponde a bajos niveles de capital social y de participación política (8). Como un bien público, el capital social explicaría por qué las sociedades con mejores niveles de calidad de vida no serían las sociedades más ricas sino aquellas más igualitarias y con alta cohesión social.

La Comisión Nacional sobre Determinantes Sociales de la Salud (CNDSS) de Brasil, en su primer informe del año 2008 (9), reconoce que el acceso a la información es un determinante social poco discutido. Desde una perspectiva macro, el concepto de brecha digital aclara la ambigüedad de las tecnologías de información y comunicación (TIC) al entender que el acceso desigual a estas conduce a inequidades en el acceso a la información. A nivel micro, el capital social, como fenómeno emergente de una red de actores y sus vínculos, es una perspectiva teórica que se abre a un contexto y discusión del concepto de acceso a la información. En ambos casos, el acceso a la información está reconocido como un determinante social de la salud y como un derecho del ciudadano. 


\section{SOBRE LA INFORMACIÓN, LA EQUIDAD Y LA SALUD}

Hablar acerca de las inequidades en los procesos de producción, distribución y uso de la información no es una novedad. La historia de la civilización humana está marcada por las diferencias, algunas injustas e innecesarias. El ideal de una sociedad interconectada por una constelación de redes de computadoras ha impreso en el concepto de la llamada sociedad de la información la expectativa, aunque sea un poco ingenua, de un mundo mejor, que sería posible gracias a la ampliación del acceso a un conjunto de bienes tangibles e intangibles.

Sin embargo, lo que es quizá inusual es que las desigualdades en la información persisten con el crecimiento en la economía globalizada de hoy. Por un lado, Internet ha acelerado el proceso de producción, circulación y consumo de información; por otro, las TIC son también las protagonistas de un proceso que profundiza las diferencias en el acceso y uso de información por parte de los individuos y la sociedad. Pero la equidad no solo se refiere al punto de vista tecnológico.

A partir de aquí, nacieron dos líneas de debate sobre el tema. Una de ellas discute la inequidad desde el concepto de equidad e información, y la otra trata la inequidad a partir de dos brechas: la digital y la de información.

La equidad es un concepto que aún requiere discusión. El concepto, derivado del griego, significa "los principios de la razón y la moderación en el ejercicio de los derechos individuales" (10). Una vez definida, valdría contestar cuál es el criterio universal de "razón y moderación" que podría definir con absoluta claridad si una situación social es justa o no; o bien, establecer si las nociones de equidad e inequidad tienen un elemento de contingencia y deben revisarse periódicamente a la luz de las condiciones sociales, económicas, políticas y culturales. La equidad es un objetivo; la inequidad, invariablemente, se crea. No son pocos los ejemplos actuales: basta recordar que algunos países, cuya cultura democrática es discutible, insisten en obstaculizar el acceso a la red mundial de computadoras y a Internet.
La literatura muestra dos tendencias epistemológicas que piensan el concepto de equidad en el campo de la información, de donde derivan las opciones políticas. La primera corriente, Ilamada vertical o jerárquica, entiende el acceso y uso de la información como función de los grupos demográficos (por ejemplo, de acuerdo al sexo, los ingresos, la edad, el idioma, el nivel educativo y la ubicación geográfica). Es decir, el acceso mayor o mejor a la información es logrado por las personas con mayor nivel social y económico. Aquí, la información es vista como un activo, una commodity consumida por la sociedad que está en mejores condiciones sociales y económicas (11). La política alternativa para una sociedad más justa sería la más amplia distribución y redistribución de bienes (por ejemplo, del apoyo financiero, de los sistemas y las fuentes de información, y de los servicios sociales) entre los diferentes grupos sociales.

La segunda perspectiva sostiene que los individuos o grupos sociales, a pesar de contar con un nivel económico y social similar, pueden ser muy distintos en función de las necesidades, el acceso y el uso de la información. Entendido como una perspectiva horizontal o heterárquica, este enfoque se centra en los intereses, conocimientos y experiencias y las contingencias de los individuos y grupos, y cómo cambian en diferentes contextos sociales y tiempos históricos. Aquí, la información es vista como un bien público intangible que es subjetivo y depende del contexto. En este caso, la calidad es mejor que la cantidad. Muchos pensadores sostienen que la equidad en el acceso y el uso de la información es una dimensión más importante para discutir políticamente que la distribución más o menos equilibrada de los bienes de información en la sociedad. Es decir, estaría implicada aquí una política que debe tener en cuenta una perspectiva subjetiva inherente a cómo la gente elige (o no) el acceso y el uso de la información.

Aunque parezca obvio que ambas perspectivas son objeto de políticas públicas, la primera dimensión de la equidad, que considera la información como una mercancía, sigue siendo la propuesta más frecuente.

Las desigualdades de la información también pueden ser discutidas a partir de un segundo razonamiento teórico que apunta hacia 
dos brechas que dividen a la sociedad: la de información y la digital. La brecha de información es heredera epistemológica del área de las Ciencias de la Información, la brecha digital es heredera de Internet y lleva inscrita en su conceptualización contribuciones de diferentes campos del conocimiento. Ambas tienen en común, tal vez, el ideal de equidad para el acceso universal.

Un panorama de la evolución histórica de estas dos tradiciones las coloca como dos líneas de investigación que van en paralelo, aun cuando deberían ser complementarias (12). El punto de partida para ambas es la afirmación de que existe una división social con respecto a los recursos en la difusión de información que, hasta los años ochenta del siglo pasado, fue un límite establecido por la comparación directa entre los países (en aquel entonces) desarrollados y en desarrollo. Con la Ilegada de Internet, desde la década del noventa, ha comenzado una elaboración más sofisticada, donde la inequidad se ha presentado, por ejemplo, bajo el concepto de "pobreza de información" (13), como una forma de privación de la información: por ausencia, abundancia o imposición.

Pero la pobreza de información no equivale a la pobreza económica: para que una persona sea "rica en información" es necesario no solo pagar por el acceso, sino tener el capital intelectual para hacer la recuperación y la evaluación de la información, y decidirse por el uso. Otros autores añaden a este punto de vista que además se necesita un conjunto de recursos políticos que puedan Ilevar a esta persona al centro de poder, que es lo que caracteriza a una persona rica en información (14).

Estas dos líneas de estudio de brechas Ilegan al siglo XXI defendiendo la misma bandera de equidad, aunque por rationales diferentes. La de la brecha de información suma en la actualidad una serie de investigaciones que apuntan hacia un mundo empobrecido que se ve privado de los recursos de información en varios niveles (político, económico, cultural y personal). Aquí, la desigualdad de la información es un concepto multifacético, que añade desventajas en el acceso, recuperación, procesamiento y uso de información.

La investigación de la brecha digital, en cambio, se centra en la brecha entre ricos y pobres en el acceso a Internet y las TIC relacionadas, incorporando un discurso sobre la importancia de la tecnología para el crecimiento económico. En consecuencia, hay una atención preferente a las implicaciones políticas y económicas de la división digital. Aquí, las palabras clave son, por ejemplo: infraestructura de la información, desarrollo de oportunidades, acceso universal En esta tradición, el concepto de brecha digital es, en la mayoría de los casos, superficial; puede ocultar agendas (para guiar la decisión política de una tecnología con la excusa de la injusticia social) y puede servir a los intereses políticos al cambiar el foco de la atención hacia las TIC y no hacia las cuestiones que las preceden (12).

A esas dos tradiciones de investigación, se añade lo que poseen de potencia real: las inequidades de información serían discutidas en un contexto de apertura interdisciplinaria, con fundamentos teóricos y tecnológicos seguros y consistentes, de carácter fundamentalmente ético, y contarían además con el apoyo explícito de sectores políticos y económicos.

Es principalmente por la ética y la universalidad que, en el caso de la salud, las inequidades de la información pueden ser vistas como un determinante importante de las inequidades en salud, en la medida en que el acceso inicuo perjudica la acción individual y colectiva de los miembros de una sociedad conspirando contra el cambio de estructuras, comportamientos y políticas. El acceso universal a la información, por lo tanto, debe ser parte del proceso de definición de políticas de salud orientadas a la equidad (3).

El informe sobre la salud mundial de 2004, World report on knowledge for better health (15), enfatiza que el acceso equitativo a la información científica, publicada y no publicada, es una acción prioritaria. Para alcanzar al conjunto de usuarios que debe beneficiarse de los resultados de las investigaciones (investigadores, tomadores de decisión, gestores del sistema de salud, profesionales de la salud, pacientes, público en general), deben ser puestas en práctica distintas estrategias de difusión de la información. En cada país, según las especificidades locales del sistema de producción de conocimiento, del sistema de salud y de la carga de enfermedad, se configuran distintas demandas de información. Incluso puede no haber demanda. Por lo tanto, es necesario informar 
sobre un acervo de conocimiento ya disponible y ponerlo a favor de la formulación de políticas públicas y de nuevas investigaciones (16).

Los debates sobre cómo actuar para reducir al mínimo las inequidades de la información continúan. En el marco teórico de los DSS, el concepto de capital social es un punto de partida para aclarar cómo, en teoría, las interacciones sociales promueven o inhiben el acceso más amplio y la circulación de la información.

\section{SOBRE EL CAPITAL SOCIAL Y LOS VÍNCULOS DE LA INFORMACIÓN}

De origen reciente, el concepto de capital social está todavía abierto: como un tema que resalta aspectos de la estructura social, o en la discusión del uso de este recurso por parte de los individuos. A nivel individual, el capital social apunta a la capacidad de relacionarse de las personas, a las redes de contactos basadas en expectativas de reciprocidad y confianza que, en conjunto, mejoran la eficiencia individual (17). En el plano colectivo, el capital social ayudaría a mantener la cohesión social, obedeciendo las normas y las leyes, la negociación en situaciones de conflicto y la prevalencia de la cooperación sobre la competencia, dando lugar a un estilo de vida basado en la asociación espontánea, en la conducta cívica, en una sociedad más abierta y democrática.

Entre las principales características del capital social señaladas en la literatura, se mencionan: fomentar la confianza social, lo que conduce a la cooperación y viceversa; facilitar la cooperación, a través de las normas de reciprocidad y las expectativas mutuas; fomentar la interacción y la interconexión de las relaciones sociales, por medio de la mejora del flujo de información y la confianza entre los individuos.

El capital social puede ser dividido en componentes cognitivos y estructurales, y cada uno de ellos, aunque sean complementarios, modela un proceso diferente de búsqueda y de comunicación de la información. El componente cognitivo incluye desde reglas, valores, actitudes y creencias hasta el acceso a las percepciones de las personas sobre el nivel de confianza, del compartir, de la reciprocidad y el intercambio interpersonal (18). Aquí se encuentran, por ejemplo, los estudios que se dedican al análisis de comportamientos de búsqueda de información (information seeking). En el campo de la salud, estos estudios traen a la superficie, por ejemplo, factores como las distinciones en la formación y escolaridad de los usuarios y orientan la elección de fuentes de información (19).

Los componentes estructurales del capital social se refieren a aspectos de la organización social, externamente observables, tales como la extensión e intensidad de las relaciones y de las actividades asociativas de la sociedad, e indicadores de sociabilidad informal y de compromiso cívico. El capital social estructural está vinculado al control de comportamientos de riesgo, la prestación de apoyo y la ayuda mutua, y los medios informales de intercambio de información; por ello se halla en el orden de las instituciones, de las políticas y de la cultura.

El primer beneficio directo del capital social es la información: el capital social facilita el acceso a fuentes de información, además de ampliar su repertorio, lo que, en teoría, lleva a la mejora de la calidad, de la relevancia y de la actualidad de la información (20). La literatura científica recoge numerosos estudios que muestran cómo las redes sociales agilizan el acceso a la información, además de hacer posible la identificación y adquisición de nuevas competencias y conocimientos en el ambiente externo. Esos estudios son particularmente importantes en el campo de la innovación donde, además del capital financiero, el capital social es lo que explicita el carácter colectivo y cooperativo del proceso de producción del conocimiento.

Los aspectos positivos del capital social son relativizados por algunos autores que identifican algunas situaciones en las que actúa mucho más como resistencia al cambio. Por ejemplo, las redes muy cohesionadas restringen más que facilitan el flujo de información. Aunque los lazos fuertes tengan beneficios claros para la información, ellos son muy dispendiosos para mantener. Una perspectiva de solidaridad muy fuerte entre los miembros internos de un grupo puede centrarlos excesivamente en la relación, reduciendo el flujo de nuevas ideas y llevando, potencialmente, al parroquialismo y 
a la inercia. Un conjunto de reglas fuertes en una comunidad puede obligar a compartir los recursos entre los miembros del grupo, lo que puede, a su vez, reducir los incentivos para la actividad innovadora y emprendedora.

De la discusión planteada hasta el momento se deduce que el concepto de "acceso a la información" exige un análisis más cuidadoso, especialmente cuando se pretende poner en la agenda de las políticas públicas.

\section{SOBRE EL CONCEPTO DE ACCESO A LA INFORMACIÓN: LÍMITES Y POTENCIAL}

En la sociedad contemporánea, la ampliación y mejora del acceso a la información son tanto una meta como un resultado esperado de la creciente utilización de las TIC. Ese carácter estratégico del acceso a la información no corresponde, sin embargo, a un campo de investigación pujante, probablemente en ningún área disciplinaria. Es un concepto que espera, todavía, una intensa discusión conceptual, metodológica y teórica (21).

El acceso a la información forma parte de un dominio más amplio de investigación de la ética de la información (22). Algunos autores delimitan el área de la ética de la información focalizando en los dilemas morales y los conflictos éticos que aparecen en las interacciones entre los seres humanos, la información (creación, organización, difusión y utilización), las TIC y los sistemas de información (22). Otros autores, a su vez, defienden que la ética de la información debería dedicarse a contestar si es o no moralmente necesario, loable o permisible negar o proporcionar a cualquiera el acceso a alguna parte de la información. Por el camino de la ética que alcanza la dimensión de la participación política es donde se discute la inequidad en el acceso a la información. Se trata, así, no solamente de la inequidad en el acceso a las ideas o de la supresión de la libertad de expresión; se trata también de la marginalización de los procesos políticos y económicos y de las actividades socioculturales (23).

Valdría, por consiguiente, preguntarse: ¿es posible intervenir en el proceso del "acceso a la información"? ¿Cómo ponerlo al servicio de una sociedad más igualitaria? ¿Cómo ponerlo al servicio de la salud? Pero, antes: ¿qué se entiende por "acceso a la información"?

Son diversas las definiciones de acceso a la información, que nacen de distintas perspectivas disciplinares, y que, se sabe, están influenciadas por innúmeros factores (por ejemplo, cognitivos, políticos, económicos, emocionales). Algunas de ellas son: "libre difusión de información", "acceso a los recursos de información y conocimiento", "libertad de información", "libertad de acceso a la información" y "derecho a tener acceso a todas las expresiones del conocimiento, la creatividad y la actividad intelectual" (21).

Seis aspectos importantes en los sistemas de información que afectan el acceso son: la identificación (o cobertura de fuentes por el sistema); la disponibilidad (facilidad de acceso, aquí incluida la mediación tecnológica y el idioma); el precio (para el usuario); el costo (para el proveedor); la comprensión; y la aceptación (24). Todos esos aspectos deberían ser entendidos principalmente en términos de capacidad y requisitos tecnológicos. Harold Innis, uno de los primeros estudiosos de las relaciones entre tecnología y sociedad, en la década del cincuenta, identificó algunas dimensiones del acceso (distancia, tiempo y control) que siguen siendo relevantes para los debates contemporáneos sobre el papel social y económico de las TIC (25).

Se realizó una revisión del concepto de acceso en seis líneas disciplinarias (biblioteconomía, ciencias de la información, sociedad de la información, comunicación de masas, comunicación organizativa y economía de la información) y se encontraron seis perspectivas distintas (11).

Tal vez, desde el área de ciencias de la información provenga la más común de las perspectivas, aquella que concibe el acceso a la información como acceso al conocimiento y sus representaciones. Aquí, se presupone el acceso a los artefactos del conocimiento (libros, artículos, películas, bases de datos, etc.) y sus representaciones (referencias y metadatos). Tener acceso al artefacto significa tener acceso al conocimiento, y se supone que ese conocimiento afecta la calidad de vida y la capacidad de toma de decisiones.

Otras dos perspectivas surgen de las investigaciones en ciencias de la información: 1) el acceso a la información como acceso a la 
tecnología, que asume que el acceso a la tecnología lleva al acceso a la información, y el dominio en el uso de la tecnología abre nuevas y numerosas fuentes de información. Emergen aquí dificultades teóricas, especialmente en la relación consecutiva entre acceso y uso. 2) el acceso a la información como acceso a la comunicación, que asume que la comprensión y uso de la información y el desarrollo de competencias llevan a la participación y toma de decisiones. Del mismo modo se espera que un nivel inicial de acceso a la información cree las competencias para el acceso y la utilización de otras fuentes de información.

Las tres últimas concepciones de acceso Ilevan a la noción de control: "el control de la participación y el control del contenido [o] sobre quién provee el acceso a qué información y con qué ventaja" (11); el acceso a la información como acceso a los bienes económicos y mercancías, lo que implica costos y beneficios; y, finalmente, acceso a la información como medio de participación, sobre todo en el proceso político, o la información como derecho y control social.

El acceso también es definido como un sistema sólido por medio del cual la información se pone a disposición de los ciudadanos (26). Ese sistema posee componentes físicos, intelectuales y sociales. De este modo, se entiende que el acceso a la información presupone un sistema de recuperación de información (una infraestructura tecnológica), que está conformado por tres dimensiones: acceso físico, acceso intelectual y acceso social. El acceso físico se refiere a la infraestructura física y electrónica donde la información está almacenada, y los caminos que deben ser recorridos para alcanzarla. Así, geografía, tecnología y economía pueden afectar el acceso físico. El acceso intelectual se refiere a la comprensión de la información contenida en el documento, incluyendo cómo está catalogada, organizada y representada. Características individuales tales como las habilidades físicas o cognitivas, la competencia lingüística y la competencia tecnológica pueden afectar el acceso intelectual. El concepto de acceso social está vinculado al capital social y sugiere que los elementos del mundo social, como las reglas sociales y las visiones del mundo, así como la propia concepción sobre la necesidad de información, intervienen en el acceso a la información. El carácter normativo del comportamiento social orientaría los mecanismos y las actividades de búsqueda de información.

En ese sentido, ninguna de las perspectivas, física o intelectual, puede responder aisladamente por el acceso a la información. Ambas son mediadas por el ambiente social de los individuos o por el capital social. La información que es accesible es aquella que está disponible en tiempo útil, es relevante, tiene un precio accesible, es fácilmente asimilable, en un idioma y un contexto que los usuarios puedan entender $y$ comprender (27).

Esta breve discusión reafirma la importancia de clarificar el concepto y las perspectivas del acceso a la información en las cuestiones relativas a la inequidad en salud. Entender las distintas dimensiones del acceso es un punto clave para la orientación en la formulación de políticas y sistemas de información. Además, el abordaje del capital social se presenta como punto de partida singular para delimitar contextos, necesidades y posibilidades del acceso a la información. Pero todavía cabe una acción complementaria que viene ocupando la agenda de discusión de los profesionales de la salud y de los educadores, denominada health literacy en la literatura científica internacional -traducida aquí como "competencias en salud"-, y que también es propuesta como tema para la agenda de investigación en DSS.

\section{CONTRIBUCIONES PARA UNA AGENDA DE PROPUESTAS}

Tal vez sea un lugar común acordar que las desigualdades económicas, sociales y políticas siempre han estado presentes en las sociedades. Quizá, resulte necesario más bien acordar que las desigualdades tienen un papel que cumplir en la dinámica del desarrollo de las sociedades, en la medida en que actúan como fuerzas impulsoras de movimientos y que tensionan la estabilidad. Si la equidad es una meta social deseable, es necesario operar con dos dimensiones de acceso a la información. La más amplia distribución de recursos, sistemas y fuentes de información debe ser complementada por la 
determinación y el compromiso político, dirigidos al fomento del capital social y de la capacitación de los individuos. El concepto de information literacy, que nació de la mano de profesionales bibliotecarios de los EE.UU. para designar habilidades vinculadas al uso de la información electrónica, es un concepto que busca expresar una condición necesaria al avance y desarrollo de la llamada sociedad del conocimiento (28), es decir, la habilidad y capacidad del individuo para identificar y acceder y hacer la mejor utilización de la información disponible en la satisfacción de sus necesidades. Dentro del campo de la salud, las competencias en salud (health literacy) se ponen al servicio de la equidad en salud.

No existe un consenso sobre la definición de competencias en salud, de la misma manera que existe poco acuerdo sobre el propio concepto de competencias. Sobreviviendo a las discusiones teóricas, lo que aquí se defiende es la necesidad de una agenda política afirmativa en lo que respecta al acceso a la información en y para la salud (29), lo que significa una estrategia de aprendizaje que provea significados y autonomía a los ciudadanos en lo que concierne a su salud individual y a la de su grupo social.

En teoría, un ciudadano competente en salud es aquel capacitado para hacer uso de los conceptos de salud e información y utilizarlos en nuevas situaciones; es aquel que toma parte en debates y diálogos públicos y privados sobre salud, medicina, conocimiento científico y cultura. Las competencias en salud son más que un estado o una condición, es un proceso, parte del complejo de las competencias humanas, y está tejida junto a dimensiones demográficas, sociopolíticas, psicosociales y culturales. En este sentido, invertir en competencias en salud es también invertir en salud (30).

\section{AGRADECIMIENTOS}

El presente artículo forma parte del Proyecto PCI-AECID (D/030704/10), dentro de la convocatoria del Programa de Cooperación Interuniversitaria e Investigación Científica $(\mathrm{PCl})$, perteneciente a las ayudas para Acciones Integradas para el Fortalecimiento Científico e Institucional, de la Agencia Española de Cooperación Internacional para el Desarrollo (AECID).

\section{CONFLICTO DE INTERESES}

Los autores declaran que no existe ningún compromiso o vínculo con las entidades financiadoras que pueda ser entendido como conflicto de intereses.

\section{REFERENCIAS BIBLIOGRÁFICAS}

1. Whitehead $M$, Burströmb B, Diderichsenb $F$. Social policies and the pathways to inequalities in health: a comparative analysis of lone mothers in Britain and Sweden. Social Science and Medicine. 2000;50(2):255-270.

2. Wing SB, Richardson D. Material living conditions and health in the United States, Canada and Western Europe: review of recent literature and bibliography. Washington: Pan American Health Organization; 1999.
3. Pellegrini Filho A. Inequidades de acceso a la información e inequidades en salud. Revista Panamericana de Salud Pública. 2002;11(56):409-412.

4. Adler N. Health disparities: monitoring, mechanism, and meaning. En: NIH Conference on understanding and reducing health disparities: contributions from the behavioral and social sciences. Bethesda. Maryland: NIH; 2006. p. 23.

5. Link B, Phelan J. Social conditions as fundamental causes of disease. Journal of Health and Social Behavior. 1995;(extra issue):80-94. 
6. Solar O, Irwin A. A conceptual framework for action on the social determinants of health: discussion paper for the Commission on Social Determinants of Health. Vancouver: $\mathrm{CSDH}$; 2007.

7. Wilkinson R. Unhealthy societies. New York: Routledge; 1997.

8. Kawachi I, Kennedy BP, Lochner K, ProthrowStith D. Social capital, income inequality, and mortality. American Journal of Public Health. 1997;87(9):1491-1498.

9. Comissão Nacional sobre Determinantes Sociais da Saúde. As causas sociais das iniqüidades em saúde no Brasil. Relatório Final. Brasil: CNDSS; 2008.

10. Lievrouw LA, Farb SE. Information and equity. Annual Review of Information Science and Technology. 2003;37:499-540.

11. McCreadie M, Rice RE. Trends in analyzing access to information. Part I: Cross-disciplinary conceptualizations of access. Information Processing and Management. 1999; 35:45-76.

12. Yu L. Understanding information inequality: making sense of the literature of the information and digital divides. Journal of Librarianship and Information Science. 2006;38:229-252.

13. Sweetland JH. Information poverty: let me count the way. Database. 1993;16(4):8-10.

14. Schiller H. Living in the number one society. Gazette: The International Journal for Communication Studies. 1998; 60(2):181-196.

15. World Health Organization. World report on knowledge for better health. Geneva: WHO; 2004.

16. Guimarães MCS. Entre o superlativo e o diminutivo: as tecnologias a serviço da difusão da informação científica. En: Mandarino ACS, Gomberg E. Leituras de novas tecnologias e saúde. Salvador: EDUFBA; 2009.

17. Coleman J. Foundations of social theories: a theory of social structure and action. Harvard: Harvard University Press; 1990. p. 300-321.

18. Islam MK, Merlo J, Kawachi I, Lindström M, Gerdtham UG. Social capital and health: does egalitarianism matter? A literature review. International Journal for Equity in Health [Internet]. 2006;5(3) [citado 10 jul 2011]. Disponible en: http://www.ncbi.nlm.nih.gov/pmc/articles/PMC15 24772/
19. Wilson TD, Allen DK, editores. Exploring the contexts of information behavior. Proceedings of the Second International Conference on Research on Information Needs, Seeking and Using in Different Contexts. Sheffield: Taylor Graham; 1998.

20. Adler PA, Seok-Woo K. Social capital: prospects for a new concept. Academy of Management Review. 2002;27(1):17-40.

21. Oltmann SM. Information access: Toward a more robust conceptualization. Proceedings of the American Society for Information Science and Technology. 2009;46(1):1-17.

22. Carbo T, Smith MM. Global information ethics: Intercultural perspectives on past and future research. Journal of the American Society for Information Science and Technology. 2008;59(7):1111-1123.

23. Reddick A. Citizens and the Internet: policy opportunities and challenges. En: Adams KG, Birdsall WF, editors. Access to information in a digital world. Ottawa: Canadian Library Association; 2004. p. 7-30.

24. Buckland M. Information and information systems. Westport: Greenwood; 1991.

25. Dutton $\mathbf{W H}$. The Internet and social transformation: reconfiguring access. En: Dutton W, Kahin B, O'Callaghan R, Wykoff A. Transforming enterprise: economic and social implications of information technology, Cambridge: MIT Press; 2005. p. 375-394.

26. Jaeger PT, Burnett G. Information access and exchange among small worlds in a democratic society: the role of policy in shaping information behavior in the post-9/11 United States. Library Quarterly. 2005;75(4):464-495.

27. Lor PJ, Britz JJ. Is a knowledge society possible without freedom of access to information? Journal of Information Science. 2007;22(4):387397.

28. Castiel L, Sanz-Valero J. El acceso a la información como determinante social de la salud. Nutrición Hospitalaria. 2010;25(supl.3):26-30.

29. Ministério da Saúde. Secretaria de Ciência, Tecnologia e Insumos Estratégicos. Departamento de Ciência e Tecnologia. Por que pesquisa em saúde? Geneva: Council on Health Research for Development, Global Forum for Health Research; 2006. 
30. Nielsen-Bohlman L, Panzer AM, Hamlin B, Kindig DA, editors. Health literacy: a prescription to end confusion. Washington: The National Academies Press; 2004.

\section{FORMA DE CITAR}

Guimarães MCS, Silva $\mathrm{CH}$, Noronha IH. El acceso a la información como determinante social de la salud. Salud Colectiva. 2011;7(Supl 1):S9-S18.

Recibido el 22 de junio de 2011

Aprobado el 10 de septiembre de 2011 